\title{
Solvability of some singular and nonsingular nonlinear third order boundary value problems
}

\author{
by D. O’REGAN* (Galway)
}

\begin{abstract}
Existence of positive solution to certain classes of singular and nonsingular third order nonlinear two point boundary value problems is examined using the idea of Topological Transversality.
\end{abstract}

1. In this paper we establish existence of positive solutions to some nonlinear singular and nonsingular third order boundary value problems of the form

$$
\left\{\begin{array}{l}
y^{\prime \prime \prime}+f\left(t, y, y^{\prime}\right)=0, \quad 0<t<1 \\
y \text { satisfies a boundary condition } B .
\end{array}\right.
$$

Here $B$ will denote either

(i) $y^{\prime \prime}(0)=c \leq 0, y^{\prime}(1)=b \geq 0, y(0)=a \geq 0$,

(ii) $y^{\prime \prime}(0)=0, y^{\prime}(0)=0, y(1)=a \geq 0$, or

(iii) $y^{\prime \prime}(0)=0, y(0)=a \geq 0, y(1)=b \geq 0$.

The above problems are singular because $f$ is allowed to be singular at $y=0$. Third order boundary value problems have become quite popular in the last ten years with most of the work concentrated on nonsingular problems; see [1], [2], [6], [7] and [8] for example. Also the author in [9] has discussed boundary value problems where $f$ is allowed to be singular at $t=0$ and $t=1$. Results for singular initial value problems may be found in [3]. This paper was motivated from [11] where S. Taliaferro considered problems of the form $y^{\prime \prime}+a(t) y^{\alpha}=0, \alpha<0$ with $y(0)=0$ and $y(1)=0$. Finally here we summarize briefly the plan of the paper. We begin by showing that (1.1) has a $C^{3}[0,1]$ solution for all $a>0$ with $a \leq a_{0}$ fixed. The key ideas used here are the Topological Transversality Theorem and the existence of a priori

1985 Mathematics Subject Classification: Primary 34B15.

* On leave from Department of Mathematics and Applied Statistics, University of Idaho, Moscow, Idaho 83843, U.S.A. 
bounds. We note that when $a>0,(1.1)$ does not involve singularities in $y$. To take care of the singular case (i.e. (1.1) with $a=0$ ) we pass to the limit in $a$ (i.e. let $a \rightarrow 0)$. Here we deduce the existence of a $C^{1}[0,1] \cap C^{3}(0,1)$ solution to (1.1) if $B$ denotes either (i) or (iii) whereas we will obtain a $C[0,1] \cap C^{3}(0,1)$ solution to (1.1) in the case where $B$ denotes (ii). The main idea in this step is the Arzelà-Ascoli Theorem.

2. We begin by establishing existence of positive solutions on $(0,1]$ to

$$
\left\{\begin{array}{l}
y^{\prime \prime \prime}+f(t, y)=0, \quad 0<t<1 \\
y^{\prime \prime}(0)=0 \\
y^{\prime}(1)=b \geq 0 \\
y(0)=a \geq 0
\end{array}\right.
$$

where $f$ satisfies the following conditions:

(2.2) $f$ is continuous on $[0,1] \times(0, \infty)$ with $\lim _{y \rightarrow 0^{+}} f(t, y)=\infty$ uniformly on compact subsets of $(0,1)$,

(2.3) $0<f(t, y) \leq g(y)$ on $[0,1] \times(0, \infty)$ where $g$ is continuous and nonincreasing on $(0, \infty)$.

In addition to the above we will have the following assumptions on $g$ :

(2.4) for any $z \in[0, \infty), G(z)=\int_{0}^{z} g(u) d u<\infty$,

(2.5) there exist constants $A>0, B>0$ and $\alpha$ with $G(z) \leq A z^{\alpha}+B$ for $z \in[0, \infty)$ and $0 \leq \alpha<2$

We will discuss separately the cases (i) $a>0$ and (ii) $a=0$. It should be remarked here that the results for the case $a>0$ will be used to discuss (2.1) with $a=0$.

Case 1: $a>0$. By a solution to (2.1) with $a>0$ we mean a function $y \in$ $C^{3}[0,1]$ that satisfies the differential equation and the boundary conditions. Also if $y$ is a solution to $(2.1)$ then $y>0$ on $(0,1)$ and so $y^{\prime \prime \prime}<0$ on $(0,1)$. Thus $y^{\prime \prime}<0$ on $(0,1)$, which in turn implies $y^{\prime}>0$ on $(0,1)$ so $y$ is strictly increasing on $(0,1)$ and in particular $y \geq a$ on $[0,1]$.

THEOREM 2.1 Suppose conditions (2.2)-(2.5) are satisfied. For $\lambda \in[0,1]$ consider the family of problems

$$
\left\{\begin{array}{l}
y^{\prime \prime \prime}+\lambda f(t, y)=0, \quad 0<t<1, \\
y^{\prime \prime}(0)=0, \quad y^{\prime}(1)=b, \quad y(0)=a .
\end{array}\right.
$$

Let $0<a \leq a_{0}$. Then there exist positive constants $M_{0}, M_{1}, M_{2}, M_{3}$ independent of $\lambda$ with $M_{0}, M_{1}$ depending on $a_{0}$ such that for $t \in[0,1]$

$a \leq y(t) \leq M_{0}, \quad b \leq y^{\prime}(t) \leq M_{1}, \quad-M_{2} \leq y^{\prime \prime}(t) \leq 0, \quad-M_{3} \leq y^{\prime \prime}(t) \leq 0$

for each solution $y$ to $\left(2.6_{\lambda}\right)$ and $a \leq a_{0}$. 
R e m a r k. It should be noted here that for fixed positive $a$, Theorem 2.1 holds if only conditions (2.2) and (2.3) are satisfied. To see this suppose $y$ is a solution to $\left(2.6_{\lambda}\right)$. Then clearly $y(t) \geq a, y^{\prime}(t) \geq b, y^{\prime \prime}(t) \leq 0, y^{\prime \prime \prime}(t) \leq 0$ for $t \in[0,1]$. The differential equation yields $-y^{\prime \prime \prime} \leq \lambda g(y) \leq g(a) \equiv M_{3}$ and integration gives $M_{2}, M_{1}$ and $M_{0}$ immediately. However, the goal of this paper is to examine singular problems and to achieve this we need to show that we can obtain $M_{0}$ and $M_{1}$ independent of $a$ for a bounded $a$. For these singular problems $a=1 / n, n \in \mathbb{N}^{+}=\{1,2, \ldots\}$. Thus we will prove Theorem 2.1 with this in mind.

Pr o of. Let $y$ be a solution to $\left(2.6_{\lambda}\right)$. The case $\lambda=0$ is trivial so assume $0<\lambda \leq 1$. Then $-y^{\prime \prime \prime} \leq \lambda g(y) \leq g(y-a)$ since $g$ is nonincreasing. Multiply by $y^{\prime}$ and integrate from 0 to $t$ to obtain

$$
-y^{\prime}(t) y^{\prime \prime}(t)+\int_{0}^{t}\left[y^{\prime \prime}(s)\right]^{2} d s \leq \int_{0}^{y(t)-a} g(u) d u=G(y(t)-a) .
$$

Thus we have $-y^{\prime}(t) y^{\prime \prime}(t) \leq G(y(t)-a)$. Here $G$ is an increasing map from $[0, \infty)$ into $[0, \infty)$ so we have $-y^{\prime}(t) y^{\prime \prime}(t) \leq G(y(t)) \leq G(y(1))$. Integrate the above inequality from $t$ to 1 to obtain

$$
-\frac{b^{2}}{2}+\frac{\left[y^{\prime}(t)\right]^{2}}{2} \leq G(y(1))
$$

so $y^{\prime}(t) \leq \sqrt{2 G(y(1))+b^{2}}$. Hence $y(1) \leq a+\sqrt{2 G(y(1))+b^{2}}$. Now assumption (2.5) implies there exists a constant $M_{0}$ (independent of $\lambda$ ) such that $y(t) \leq y(1) \leq M_{0}$ for $t \in[0,1]$. In addition the above analysis yields

$$
y^{\prime}(t) \leq \sqrt{2 G(y(1))+b^{2}} \leq \sqrt{2 G\left(M_{0}\right)+b^{2}}=M_{1} .
$$

If $a \leq a_{0}$ then we can choose $M_{0}$ and $M_{1}$ independent of $a$ since $y(1) \leq$ $a_{0}+\sqrt{2 G(y(1))+b^{2}}$.

Also we have $0 \leq-y^{\prime \prime \prime}(t) \leq \lambda \sup _{\left[a, M_{0}\right]} g(u) \leq g(a)=M_{2}$ and finally integration from 0 to $t$ yields a bound for $y^{\prime \prime}(t)$.

We will now apply the Topological Transversality Theorem [4, 5] to obtain our basic existence theorem. For notational purposes set

$$
\begin{aligned}
C_{B}^{3}[0,1] & =\left\{u \in C^{3}[0,1] ; u(0)=a, u^{\prime}(1)=b, u^{\prime \prime}(0)=0\right\}, \\
C_{B_{0}}^{3}[0,1] & =\left\{u \in C^{3}[0,1] ; u(0)=0, u^{\prime}(1)=0, u^{\prime \prime}(0)=0\right\} .
\end{aligned}
$$

ThEOREM 2.2. Suppose conditions (2.2) and (2.3) are satisfied. Then a $C^{3}[0,1]$ solution of $(2.1)$ exists.

Pr o of. Consider the family of problems

$$
\left\{\begin{array}{l}
y^{\prime \prime \prime}+\lambda \bar{f}(t, y)=0, \quad 0<\lambda<1, \\
y^{\prime \prime}(0)=0, \quad y^{\prime}(1)=b, \quad y(0)=a,
\end{array}\right.
$$


where

$$
\bar{f}(t, y)= \begin{cases}f(t, y) & \text { for } y \geq a \\ f(t, a) & \text { for } y \leq a\end{cases}
$$

Every solution $v$ of $\left(2.7_{\lambda}\right)$ satisfies $v \geq a$ and hence is a solution to $\left(2.6_{\lambda}\right)$. Also $\bar{f}$ satisfies the hypothesis imposed on $f$ since for $0<y \leq a, \bar{f}(t, y)=$ $f(t, a) \leq g(a) \leq g(y)$. Hence the conclusion of Theorem 2.1 remains valid for solutions to $\left(2.7_{\lambda}\right)$. Let $\tilde{M}_{0}=\max \left\{M_{0}, b+a\right\}$ and

$$
\begin{aligned}
V=\left\{u \in C_{B}^{3}[0,1]: a / 2<u(t)<\right. & \tilde{M}_{0}+1,\left|u^{\prime}(t)\right|<M_{1}, \\
& \left.\left|u^{\prime \prime}(t)\right|<M_{2}+1,\left|u^{\prime \prime \prime}(t)\right|<M_{3}+1\right\} .
\end{aligned}
$$

$V$ is an open subset of $C_{B}^{3}[0,1]$ which in turn is a convex subset of $C^{3}[0,1]$.

Define mappings $F_{\lambda}: C[0,1] \rightarrow C[0,1], j: C_{B}^{3}[0,1] \rightarrow C[0,1], L:$ $C_{B}^{3}[0,1] \rightarrow C[0,1]$ by $\left(F_{\lambda} u\right)(t)=-\lambda \bar{f}(t, u(t)), j u=u$ and $L u(t)=u^{\prime \prime \prime}(t)$. $F_{\lambda}$ is continuous and $j$ is completely continuous by the Arzelà-Ascoli Theorem. Finally, we claim $L^{-1}$ exists and is continuous. To see this define $N: C_{B_{0}}^{3}[0,1] \rightarrow C[0,1]$ by $N u=u^{\prime \prime \prime} . N^{-1}$ is a continuous linear operator by the Bounded Inverse Theorem; see [10, Theorem 5.10]. Thus $L^{-1}$ exists and is given by

$$
\left(L^{-1} g\right)(x)=b x+a+\left(N^{-1} g\right)(x)
$$

and so is continuous. Now define the map $H_{\lambda}: \bar{V} \rightarrow C_{B}^{3}[0,1]$ by $H_{\lambda} u=$ $L^{-1} F_{\lambda} j u$. $H_{\lambda}$ is a compact homotopy and $H_{\lambda} u=u$ means $L u=F_{\lambda} j u$, i.e. $u^{\prime \prime \prime}=-\lambda \bar{f}(t, u(t))$ and $u$ satisfies the boundary conditions. Therefore $H_{\lambda}$ is fixed point free on $\partial V$ by construction of $V$ and Theorem 2.1. Finally, for any $u \in \bar{V}, H_{0}(u)=u_{0}, u_{0}(x)=b x+a$, i.e. $H_{0}$ is a constant map and so is essential [4]. The topological transversality theorem [4. p. 86] implies that $H_{1}$ is essential, i.e. $\left(2.7_{1}\right)$ has a solution and therefore (2.1) has a solution.

It is also possible to consider in this section problems of the form

$$
\left\{\begin{array}{l}
y^{\prime \prime \prime}+f\left(t, y, y^{\prime}\right)=0, \quad 0<t<1 \\
y^{\prime \prime}(0)=c \leq 0 \\
y^{\prime}(1)=b>0 \\
y(0)=a>0
\end{array}\right.
$$

where $f$ satisfies the following:

$f$ is continuous on $[0,1] \times(0, \infty) \times(-\infty, \infty)$ with $\lim _{y \rightarrow 0^{+}} f(t, y, p)$ $=\infty$ uniformly on compact subsets of $[0,1] \times(-\infty, \infty) \backslash\{0\}$,

$0<f(t, y, p) \leq g(y) p$ on $[0,1] \times(0, \infty) \times[b, \infty)$ where $g$ is continuous and nonincreasing on $(0, \infty)$ with $f \geq 0$ on $[0,1] \times(0, \infty) \times$ $(-\infty, \infty)$. 
THEOREM 2.3. Suppose that conditions (2.9), (2.10), (2.4) and (2.5) with $0 \leq \alpha<1$ are satisfied. For $\lambda \in[0,1]$ consider

$$
\left\{\begin{array}{l}
y^{\prime \prime \prime}+\lambda f\left(t, y, y^{\prime}\right)=0, \quad 0<t<1 \\
y^{\prime \prime}(0)=c, y^{\prime}(1)=b, \quad y(0)=a
\end{array}\right.
$$

Then there exists constants $M_{0}, M_{1}, M_{2}, M_{3}$ independent of $\lambda$ such that for $t \in[0,1]$

$$
a \leq y(t) \leq M_{0}, b \leq y(t) \leq M_{1},-M_{2} \leq y^{\prime \prime}(t) \leq c,-M_{3} \leq y^{\prime \prime \prime}(t) \leq 0
$$

for each solution $y$ to $\left(2.11_{\lambda}\right)$.

Proof. Suppose $y$ is a solution to $\left(2.11_{\lambda}\right)$. Assume $0<\lambda \leq 1$. Clearly (since (2.9) and (2.10) are satisfied) $y(t) \geq a, y^{\prime}(t) \geq b, y^{\prime \prime}(t) \leq c, y^{\prime \prime \prime}(t) \leq 0$ for $t \in[0,1]$. We also have $-y^{\prime \prime \prime} \geq g(y-a) y^{\prime}$ since $g$ is nonincreasing. Integrating from 0 to $t$ yields $-y^{\prime \prime}(t) \leq G(y(t)-a)-c \leq G(y(t))-c$ since $G$ is an increasing map from $[0, \infty)$ into $[0, \infty)$. Now integrate from $t$ to 1 to obtain

$$
y^{\prime}(t) \leq \int_{t}^{1} G(y(u)) d u+b-c \leq G(y(1))+b-c
$$

and so $y(1) \leq G(y(1))+b+a-c$. Assumption (2.5) with $0 \leq \alpha<1$ implies there exists a constant $M_{0}$ (independent of $\lambda$ ) such that $y(1) \leq M_{0}$. In addition the above analysis yields $y^{\prime}(t) \leq G\left(M_{0}\right)+b-c=M_{1}$ and $-y^{\prime \prime}(t) \leq G(y(t))-c \leq G\left(M_{0}\right)-c=M_{2}-c$.

Remark. If $a \leq a_{0}$ then $M_{0}, M_{1}$, and $M_{2}$ can be chosen independent of $a$.

Finally, we have $0 \leq-y^{\prime \prime \prime}(t) \leq \lambda \sup _{\left[a, M_{0}\right]} g(y) \sup _{\left[b, M_{1}\right]} y^{\prime}=M_{3}$.

THEOREM 2.4. Suppose that conditions (2.9), (2.10), (2.4) and (2.5) with $0 \leq \alpha<1$ are satisfied. Then a $C^{3}[0,1]$ solution of $(2.8)$ exists.

Proof. This follows the reasoning in Theorem 2.2. The only change is to define

$$
\bar{f}(t, y, p)= \begin{cases}f(t, y, p) & \text { for } y \geq a, \\ f(t, a, p) & \text { for } y \leq a,\end{cases}
$$

and here $F_{\lambda}: C^{1}[0,1] \rightarrow C[0,1], j: C_{B}^{3}[0,1] \rightarrow C^{1}[0,1]$. Of course $j u=u$ and $\left(F_{\lambda} u\right)(t)=-\lambda \bar{f}\left(t, u(t), u^{\prime}(t)\right)$

Case 2: $a=0$. When $a=0$, a solution to (2.1) will mean a function

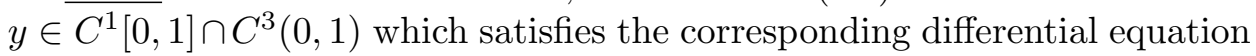
and boundary conditions.

THEOREM 2.5. Suppose conditions (2.2)-(2.5) are satisfied. In addition suppose $f$ satisfies: 
(2.12) For each constant $M>0$ there exists $\psi(t)$ continuous on $[0,1]$ and positive on $(0,1)$ such that $f(t, y) \geq \psi(t)$ on $[0,1] \times(0, M]$,

$$
\left\{\begin{array}{l}
\int_{0}^{1} g(\theta(t)) d t<\infty \text { where } \theta(t)=b t, b>0 \text { and for } b=0 \\
\theta(t)=\int_{0}^{t}\left(t-.5 t^{2}-.5 s^{2}\right) \psi(s) d s+\int_{t}^{1}(1-s) t \psi(s) d s
\end{array}\right.
$$

Then a $C^{1}[0,1] \cap C^{3}(0,1)$ solution of $(2.1)$ exists.

Proof. We look at the family of problems (with $n \in \mathbb{N}^{+}$)

$$
\left\{\begin{array}{l}
y^{\prime \prime \prime}+f(t, y)=0, \quad 0<t<1 \\
y(0)=1 / n \\
y^{\prime}(1)=b \\
y^{\prime \prime}(0)=0
\end{array}\right.
$$

Theorem 2.2 implies that $\left(2.13_{n}\right)$ has a solution $y_{n}$ for each $n$. Moreover, there are constants $M_{0}$ and $M_{1}$ independent of $n$ (set $a_{0}=1$ in Theorem 2.1) such that $1 / n \leq y_{n}(t) \leq M_{0},\left|y_{n}^{\prime}(t)\right| \leq M_{1}$ for $t \in[0,1]$. In addition we claim that there is a constant $M_{2}$ independent of $n$ such that $\left\|y_{n}^{\prime \prime}\right\|_{L^{2}} \leq M_{2}$; to see this notice $-y_{n}^{\prime} y_{n}^{\prime \prime \prime} \leq g\left(y_{n}\right) y_{n}^{\prime}$ and integration from 0 to 1 yields

$$
-b y_{n}^{\prime \prime}(1)+\int_{0}^{1}\left[y_{n}^{\prime \prime}(s)\right]^{2} d s \leq \int_{0}^{M_{0}} g(u) d u .
$$

Since $y_{n}^{\prime \prime}(1) \leq 0$ our claim is established. The Arzelà-Ascoli Theorem guarantees the existence of a subsequence $y_{n^{\prime}}$ converging uniformly on $[0,1]$ to some function $y \in C^{1}[0,1]$. Clearly $y \geq 0$ on $[0,1]$ with $y(0)=a$ and $y^{\prime}(1)=b$. In fact $y>0$ on $(0,1] ;-y_{n}^{\prime \prime \prime}(t) \geq \psi(t)$ so triple integration yields

$$
\begin{aligned}
y_{n}(t) \geq & 1 / n+b t+\int_{0}^{t} \psi(u)\left[\int_{u}^{t}(1-s) d s\right] d u \\
& +\int_{0}^{t} u(1-u) \psi(u) d u+\int_{t}^{1} t(1-u) \psi d u .
\end{aligned}
$$

Now $y_{n}^{\prime}$ satisfies the integral equation

$$
\begin{gathered}
y_{n^{\prime}}(t)=y_{n^{\prime}}(1)-b(t-1)+\frac{y_{n^{\prime}}^{\prime \prime}(1)}{2}(t-1)^{2} \\
-\int_{t}^{1}(s-t)^{2} f\left(s, y_{n^{\prime}}(s)\right) d s .
\end{gathered}
$$

For $t \in(0,1]$ we have $f\left(s, y_{n^{\prime}}(s)\right) \rightarrow f(s, y(s))$ uniformly in $s \in[t, 1]$ since $f$ is uniformly continuous on compact subsets of $[0,1] \times\left(0, M_{0}\right]$. Thus letting 
$n^{\prime} \rightarrow \infty$ yields (with $q \in \mathbb{R}$ )

$$
y(t)=y(1)-b(t-1)+q \frac{(t-1)^{2}}{2}-\int_{t}^{1}(s-t)^{2} f(s, y(s)) d s .
$$

From the integral equation we see that $y^{\prime \prime \prime}=-f(t, y)$ and $\left(2.12^{*}\right)$ together with the Lebesgue dominated convergence theorem implies

$$
\begin{aligned}
0=\lim _{n^{\prime} \rightarrow \infty} y_{n^{\prime}}^{\prime \prime}(0) & =\lim _{n^{\prime} \rightarrow \infty}\left[\int_{0}^{1} f\left(s, y_{n^{\prime}}(s)\right) d s-y_{n^{\prime}}^{\prime \prime}(1)\right] \\
& =\int_{0}^{1} f(s, y(s)) d s-q=y^{\prime \prime}(0)
\end{aligned}
$$

since $f\left(t, y_{n^{\prime}}(t)\right) \leq g\left(y_{n^{\prime}}(t)\right) \leq g(\theta(t)) \in L^{1}$; noting of course that $-y_{n^{\prime}}^{\prime \prime \prime} \geq$ $\psi(t)$ implies $y_{n^{\prime}}(t) \geq \theta(t)$ if $b=0$ whereas $y_{n^{\prime}}(t) \geq b t$ if $b>0$.

R e mark. If $b>0$, assumption (2.12) can be removed in the statement of Theorem 2.5 and existence of a solution to (2.1) is guaranteed.

We can obtain a similar result for problems of the form (2.8).

TheOREM 2.6. Suppose (2.9), (2.10), (2.4), (2.12*) with $b>0$, and (2.5) with $0 \leq \alpha<1$ are satisfied. Then a $C^{1}[0,1] \cap C^{3}(0,1)$ solution of $(2.8)$ exists.

Proof. Examine the problems (with $n \in \mathbb{N}^{+}$)

$$
\left\{\begin{array}{l}
y^{\prime \prime \prime}+f\left(t, y, y^{\prime}\right)=0, \quad 0<t<1, \\
y(0)=1 / n \\
y^{\prime}(1)=b \\
y^{\prime \prime}(0)=c
\end{array}\right.
$$

Theorem 2.4 guarantees that $\left(2.14_{n}\right)$ has a solution $y_{n}$ for each $n$ and also there exist constants $M_{0}, M_{1}$ and $M_{2}$ independent of $n$ such that $1 / n \leq$ $y_{n}(t) \leq M_{0}, b \leq y_{n}^{\prime}(t) \leq M_{1},\left|y_{n}^{\prime \prime}(t)\right| \leq M_{2}$ for $t \in[0,1]$. Consequently, we have a subsequence $y_{n^{\prime}}$ converging uniformly on $[0,1]$ to some function $y \in C^{1}[0,1]$ and in addition $y \geq 0, y^{\prime} \geq b$ on $[0,1]$ with $y(0)=0$ and $y^{\prime}(1)=b$. Since $b>0$ we have $y>0$ on $(0,1]$. Moreover, $y_{n^{\prime}}$ satisfies

$$
\begin{aligned}
y_{n^{\prime}}(t)= & y_{n^{\prime}}(1)-b(t-1)+\frac{y_{n^{\prime}}^{\prime \prime}(1)}{2}(t-1)^{2} \\
& -\int_{t}^{1}(s-t)^{2} f\left(s, y_{n^{\prime}}(s), y_{n^{\prime}}^{\prime}(s)\right) d s
\end{aligned}
$$

so for $t \in(0,1]$ and $s \in[t, 1]$ we have $f\left(s, y_{n^{\prime}}(s), y_{n^{\prime}}^{\prime}(s)\right) \rightarrow f\left(s, y(s), y^{\prime}(s)\right)$ uniformly since $f$ is uniformly continuous on compact subsets of $[0,1] \times$ 
$\left(0, M_{0}\right] \times\left[b, M_{1}\right]$. The result follows by allowing $n^{\prime} \rightarrow \infty$.

3. In this section we give a brief treatment to the problem of obtaining positive solutions on $[0,1)$ to

$$
\left\{\begin{array}{l}
y^{\prime \prime \prime}+f(t, y)=0, \quad 0<t<1 \\
y^{\prime \prime}(0)=0 \\
y^{\prime}(0)=0 \\
y(1)=a \geq 0
\end{array}\right.
$$

Case 1: $a>0$. Now if $y \in C^{3}[0,1]$ is a solution to (3.1) then $y^{\prime \prime \prime}<0$ on $(0,1)$ so $y^{\prime \prime}<0$ on $(0,1)$, which in turn implies $y^{\prime}<0$ on $(0,1)$ so $y$ is strictly decreasing on $(0,1)$ and in particular $y \geq a$ on $[0,1]$.

THEOREM 3.1. Suppose $f$ satisfies conditions (2.2) and (2.3). For $\lambda \in$ $[0,1]$ consider

$$
\left\{\begin{array}{l}
y^{\prime \prime \prime}+\lambda f(t, y)=0, \quad 0<t<1 \\
y^{\prime \prime}(0)=0, \quad y^{\prime}(0)=0, \quad y(1)=a
\end{array}\right.
$$

Then there exist positive constants $M_{0}, M_{1}, M_{2}$ independent of $\lambda$ such that for $t \in[0,1]$

$$
a \leq y(t) \leq M_{0},-M_{1} \leq y^{\prime}(t) \leq 0,-M_{2} \leq y^{\prime \prime}(t) \leq 0,-M_{2} \leq y^{\prime \prime \prime}(t) \leq 0
$$

for each solution $y$ to $\left(3.2_{\lambda}\right)$.

Proof. Let $0<\lambda \leq 1$. For solutions $y$ to $\left(3.2_{\lambda}\right),-y^{\prime \prime \prime} \leq g(y)$ so integration from 0 to $t$ yields

$$
-y^{\prime \prime}(t) \leq \int_{0}^{t} g(y(u)) d u \leq g(y(t)) \leq g(y(t)-a)
$$

since $g$ is nonincreasing. Another integration from 0 to $t$ will give $-y^{\prime}(t) \leq$ $g(y(t)-a)$; finally, integrate the above inequality from $t$ to 1 to obtain

$$
\int_{0}^{y(t)-a} \frac{d u}{g(u)} \leq 1
$$

Since $1 / g$ is nondecreasing and $\int_{0}^{z} d u / g(u)=\infty, G$ is an increasing map from $[0, \infty)$ onto $[0, \infty)$ and therefore has an increasing inverse $G^{-1}$. Thus we have $y(t) \leq G^{-1}(1)+a=M_{0}$ for $t \in[0,1]$. Returning to $-y^{\prime \prime}(t) \leq g(y(t))$ and multiplying by $y^{\prime}$ we obtain $-y^{\prime} y^{\prime \prime} \geq g(y) y^{\prime}$. Integrating from 0 to $t$ yields

$$
-\frac{\left[y^{\prime}(t)\right]^{2}}{2} \geq \int_{y(0)}^{y(t)} g(u) d u=-\int_{y(t)}^{y(0)} g(u) d u
$$


so

$$
\left[y^{\prime}(t)\right]^{2} \leq 2 \int_{a}^{M_{0}} g(u) d u .
$$

Thus we have $-M_{1} \leq y^{\prime}(t) \leq 0$.

Remark. If $a \leq a_{0}$ and $\int_{0}^{M_{0}} g(u) d u<\infty$ then it is possible to obtain $M_{0}$ and $M_{1}$ independent of $a$.

In addition, $0 \leq-y^{\prime \prime \prime}(t) \leq \sup _{\left[a, M_{0}\right]} g(y) \equiv M_{2}$ and integration yields the bound for $y^{\prime \prime}(t)$.

Essentially the same reasoning as in Theorem 2.2 establishes

TheOREM 3.2. Suppose $f$ satisfies (2.2) and (2.3). Then a $C^{3}[0,1]$ solution to (3.1) exists.

Case 2: $a=0$

THEOREM 3.3. Suppose $f$ satisfies (2.2), (2.3) and (2.12). In addition, suppose $g$ satisfies (2.4). Then a $C[0,1] \cap C^{3}(0,1)$ solution to (3.1) exists.

Proof. This follows from a slight (and easy) modification of the proof of Theorem 2.5.

4. Finally, to conclude the paper we establish existence of positive solutions on $(0,1)$ to

$$
\left\{\begin{array}{l}
y^{\prime \prime \prime}+f(t, y)=0, \quad 0<t<1 \\
y^{\prime \prime}(0)=0 \\
y(0)=a \geq 0 \\
y(1)=b \geq 0
\end{array}\right.
$$

where we assume without loss of generality that $a \leq b$.

$\underline{\text { Case } 1: ~} a>0$. Suppose $y \in C^{3}[0,1]$ is any solution to (4.1). Then $y>0$ on $(0,1)$ and so $y^{\prime \prime \prime}<0$. Hence $y^{\prime \prime}<0$ on $(0,1)$, which in turn implies $y^{\prime}$ is strictly decreasing on $(0,1)$ and of course $y \geq a$ on $[0,1]$.

Theorem 4.1. Suppose $f$ satisfies (2.2), (2.3) and $g$ satisfies (2.4) and (2.5). In addition, suppose $g$ satisfies the following:

(4.2) $y g(y)$ is nondecreasing on $(0, \infty)$.

For $\lambda \in[0,1]$ consider

$$
\left\{\begin{array}{l}
y^{\prime \prime \prime}+\lambda f(t, y)=0, \quad 0<t<1 \\
y^{\prime \prime}(0)=0, \quad y(0)=a, \quad y(1)=b .
\end{array}\right.
$$

Then there exist constants $M_{0}, M_{1}, M_{2}$ independent of $\lambda$ such that

$$
a \leq y(t) \leq M_{0}, \quad\left|y^{\prime}(t)\right| \leq M_{1}, \quad-M_{2} \leq y^{\prime \prime}(t) \leq 0, \quad-M_{2} \leq y^{\prime \prime \prime}(t) \leq 0
$$


for each solution y to $\left(4.3_{\lambda}\right)$.

Remark. Again it should be noted that Theorem 4.1 is true if only conditions (2.2) and (2.3) are satisfied. However in hindsight we need to obtain $M_{0}$ and $M_{1}$ independent of $n$ if $a=1 / n$ and/or $b=1 / n, n \in \mathbb{N}^{+}$.

Proof. Suppose $0<\lambda \leq 1$. Let $y$ be a solution to $\left(4.3_{\lambda}\right)$ and $y_{\max }$ the maximum of $y(t)$ on $[0,1]$. If the maximum occurs at the end points then $y_{\max } \leq b$. On the other hand, suppose $y_{\max }$ occurs at $t_{0} \in(0,1)$, so $y^{\prime}\left(t_{0}\right)=0$. Now for $t \leq t_{0}$ we have $-y^{\prime} y^{\prime \prime \prime} \leq g(y) y^{\prime} \leq g(y-a) y^{\prime}$ so integrating from 0 to $t$ yields

$$
-y^{\prime}(t) y^{\prime \prime}(t)+\int_{0}^{t}\left[y^{\prime \prime}(s)\right]^{2} d s \leq \int_{0}^{y(t)-a} g(u) d u=G(y(t)-a) .
$$

Thus $-y^{\prime}(t) y^{\prime \prime}(t) \leq G\left(y\left(t_{0}\right)\right)$ for $t \leq t_{0}$ and integration from $t$ to $t_{0}$ will give

$$
\frac{\left[y^{\prime}(t)\right]^{2}}{2} \leq G\left(y\left(t_{0}\right)\right) \text {. }
$$

Hence $y^{\prime}(t) \leq \sqrt{2 G\left(y\left(t_{0}\right)\right)}$, which in turn yields $y\left(t_{0}\right) \leq \sqrt{2 G\left(y\left(t_{0}\right)\right)}+a$. Consequently, there exists a constant $K_{0}$ (independent of $\lambda$ ) such that $y\left(t_{0}\right) \leq K_{0}$. This implies $a \leq y(t) \leq M_{0}=\max \left\{K_{0}, b\right\}$.

Rem ark. If $a=1 / n$ and/or $b=1 / n$ where $n \in \mathbb{N}^{+}$we can choose $M_{0}$ independent of $n$.

To find $M_{1}$ there are two cases to consider; either (A) there exists $t_{0} \in$ $(0,1)$ with $y^{\prime}\left(t_{0}\right)=0$ or $(\mathrm{B}) y^{\prime}>0$ on $(0,1)$.

Case $\mathrm{A}$. The above analysis yields for $t \leq t_{0}$

$$
0 \leq y^{\prime}(t) \leq \sqrt{2 G\left(y\left(t_{0}\right)\right)} \leq \sqrt{2 \int_{0}^{M_{0}} g(u) d u}=K_{1} .
$$

In addition we also have for $t \leq t_{0}$

$$
-y(t) y^{\prime \prime \prime}(t) \leq y(t) g(y(t)) \leq y\left(t_{0}\right) g\left(y\left(t_{0}\right)\right) \leq M_{0} g\left(M_{0}\right)=K_{2}
$$

since $g$ satisfies (4.2). Integration from 0 to $t_{0}$ now gives

$$
-y\left(t_{0}\right) y^{\prime \prime}\left(t_{0}\right)+\int_{0}^{t_{0}} y^{\prime}(s) y^{\prime \prime}(s) d s \leq K_{2}
$$

and so

$$
-y\left(t_{0}\right) y^{\prime \prime}\left(t_{0}\right) \leq K_{2}+\frac{\left[y^{\prime}(0)\right]^{2}}{2} \leq K_{2}+\frac{K_{1}^{2}}{2}=K_{3} .
$$


Hence $-y\left(t_{0}\right) y^{\prime \prime}\left(t_{0}\right) \leq K_{3}$. For the case $t \geq t_{0}$ we have $-y(t) y^{\prime \prime \prime}(t) \leq$ $y(t) g(y(t)) \leq K_{2}$ so integrating from $t_{0}$ to 1 yields

$$
y\left(t_{0}\right) y^{\prime \prime}\left(t_{0}\right)-y(1) y^{\prime \prime}(1)+\frac{\left[y^{\prime}(1)\right]^{2}}{2} \leq K_{2}
$$

from which we deduce

$$
\left[y^{\prime}(1)\right]^{2} \leq 2 K_{2}+2 K_{3} \quad \text { since } y(1) y^{\prime \prime}(1) \leq 0 .
$$

Thus there exists a constant $K_{4}>0$ such that $0 \leq-y^{\prime}(1) \leq K_{4}$ and consequently $0 \leq-y^{\prime}(t) \leq K_{4}$ for $t \geq t_{0}$.

Case B: $y^{\prime}>0$ on $(0,1)$. Since $y^{\prime \prime}<0, y^{\prime}$ is decreasing on $(0,1)$. The mean value theorem implies there exists $\eta \in(0,1)$ with $y^{\prime}(\eta)=b-a$. For $t \geq \eta, 0 \leq y^{\prime}(t) \leq b-a \leq b$. However, for $t<\eta$ we have $y^{\prime}(t) \geq b-a$ and $-y^{\prime}(t) y^{\prime \prime \prime}(t) \leq g(y) y^{\prime}$. Integrate from 0 to $t<\eta$ to obtain $-y^{\prime}(t) y^{\prime \prime \prime}(t) \leq$ $\int_{0}^{y(\eta)} g(u) d u$ and then

$$
\frac{\left[y^{\prime}(t)\right]^{2}}{2} \leq \int_{0}^{y(\eta)} g(u) d u+\frac{\left[y^{\prime}(\eta)\right]^{2}}{2} \leq \int_{0}^{M_{0}} g(u) d u+\frac{(b-a)^{2}}{2}
$$

Combining both cases implies that we have shown $\left|y^{\prime}(t)\right| \leq M_{1}$ for any solution $y$ to $\left(4.3_{\lambda}\right)$.

R e mark. Once again if $a=1 / n$ and/or $b=1 / n$ then it is possible to choose $M_{1}$ independent of $n$.

To complete the proof note that $0 \leq-y^{\prime \prime \prime}(t) \leq \lambda \sup _{\left[a, M_{0}\right]} g(u) \equiv M_{2}$ and integration yields the bound for $y^{\prime \prime}$.

The reasoning in Theorem 2.2 immediately yields

THEOREM 4.2. Suppose conditions (2.2) and (2.3) are satisfied. Then a $C^{3}[0,1]$ solution of (4.1) exists.

Case 2: $a=0$

THEOREM 4.3. Suppose conditions (2.2)-(2.5), (2.12) and (4.2) are satisfied. In addition, suppose $\left(2.12^{*}\right)$ holds with

$$
\theta(t)=-\int_{0}^{t}(t-z) \int_{0}^{z} \psi(s) d s d z+t \int_{0}^{1}(1-z) \int_{0}^{z} \psi(s) d s d z
$$

Then a $C^{1}[0,1] \cap C^{3}(0,1)$ solution of $(4.1)$ exists.

Proof. This follows almost verbatim the ideas in Theorem 2.5. Here if $b>0$ consider the family of problems

$$
\left\{\begin{array}{l}
y^{\prime \prime \prime}+f(t, y)=0, \quad 0<t<1 \\
y(0)=1 / n, \quad y(1)=b, \quad y^{\prime \prime}(0)=0,
\end{array}\right.
$$


whereas if $b=0$ consider

$$
\left\{\begin{array}{l}
y^{\prime \prime \prime}+f(t, y)=0, \quad 0<t<1 \\
y(0)=1 / n, \quad y(1)=1 / n, \quad y^{\prime \prime}(0)=0
\end{array}\right.
$$

\section{References}

[1] R. P. Agarwal, Some new results on two-point problems for higher order differential equations, Funkc. Ekvac. 29 (1986), 197-212.

[2] —, Existence-uniqueness and iterative methods for third-order boundary value problems, J. Comput. Appl. Math. 17 (1987), 271-289.

[3] L. E. Bobisud and D. O'Regan, Existence of solutions to some singular initial value problems, J. Math. Anal. Appl. 133 (1988), 214-230.

[4] J. Dugundji and A. Granas, Fixed Point Theory, Vol. 1, Monograf. Mat. 61, PWN, Warszawa 1982.

[5] A. Granas, R. B. Guenther and J. W. Lee, Nonlinear boundary value problems for ordinary differential equations, Dissertationes Math. 244 (1985).

[6] - -,-, Nonlinear boundary value problems for some classes of ordinary differential equations, Rocky Mountain J. Math. 10 (1980), 35-58.

[7] L. K. Jackson, Existence and uniqueness of solutions of boundary value problems for Lipschitz equations, J. Differential Equations 32 (1979), 76-90.

[8] D. O'Regan, Topological transversality: Applications to third order boundary value problems, SIAM J. Math. Anal. 18 (3) (1987), 630-641.

[9] - Singular and nonsingular third order boundary value problems, Proc. Royal Irish Acad. 90A (1990), 29-42.

[10] W. Rudin, Real and Complex Analysis, McGraw-Hill, New York 1966.

[11] S. D. Taliaferro, A nonlinear singular boundary value problem, Nonlinear Anal. 3 (1979), 897-904.

MATHEMATICS DEPARTMENT

UNIVERSITY COLLEGE GALWAY

NATIONAL UNIVERSITY OF IRELAND

GALWAY, IRELAND

Reçu par la Rédaction le 5.4.1990

Révisé le 1.8.1990 